\title{
Ceramides and sphingosine-1-phosphate mediate the distinct effects of M1/M2-macrophage infusion on liver recovery after hepatectomy
}

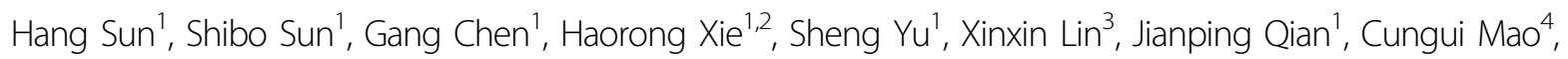
Hongxian Peng ${ }^{1}$, Hao Chen ${ }^{1}$, Xuefang Chen ${ }^{1,2}$, Yiyi Li ${ }^{5}$, Cuiting Liu' ${ }^{6}$, Junmin Shi ${ }^{6}$, Bili Zhu' ${ }^{7}$, Linghong Guo ${ }^{1,8}$,

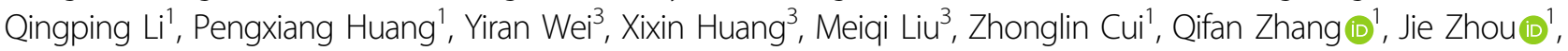
Chuanjiang Li (i) ${ }^{1}$ and Kai Wang (1)

\begin{abstract}
Post-hepatectomy liver dysfunction is a life-threatening morbidity that lacks efficient therapy. Bioactive lipids involved in macrophage polarization crucially regulate tissue injury and regeneration. Herein, we investigate the key bioactive lipids that mediate the cytotherapeutic potential of polarized-macrophage for post-hepatectomy liver dysfunction. Untargeted lipidomics identified elevation of ceramide (CER) metabolites as signature lipid species relevant to M1/M2 polarization in mouse bone-marrow-derived-macrophages (BMDMs). M1 BMDMs expressed a CER-generationmetabolic pattern, leading to elevation of CER; M2 BMDMs expressed a CER-breakdown-metabolic pattern, resulting in upregulation of sphingosine-1-phosphate (S1P). After infusing M1- or M2-polarized BMDMs into the mouse liver after hepatectomy, we found that M1-BMDM infusion increased M1 polarization and CER accumulation, resulting in exaggeration of hepatocyte apoptosis and liver dysfunction. Conversely, M2-BMDM infusion enhanced M2 polarization and S1P generation, leading to alleviation of liver dysfunction with improved hepatocyte proliferation. Treatment of exogenous CER and S1P or inhibition CER and S1P synthesis by siRNA targeting relevant enzymes further revealed that CER induced apoptosis while S1P promoted proliferation in post-hepatectomy primary hepatocytes. In conclusion, CER and S1P are uncovered as critical lipid mediators for M1- and M2-polarized BMDMs to promote injury and regeneration in the liver after hepatectomy, respectively. Notably, the upregulation of hepatic S1P induced by M2-BMDM infusion may have therapeutic potential for post-hepatectomy liver dysfunction.
\end{abstract}

Correspondence: Qifan Zhang (zhangqifansmu@163.com) or Jie Zhou (jacky@smu.edu.cn) or Chuanjiang Li (licj@smu.edu.cn) or Kai Wang (kaiwang@smu.edu.cn)

'Division of Hepatobiliopancreatic Surgery, Department of General Surgery, Nanfang Hospital, Southern Medical University, Guangzhou, Guangdong, China

${ }^{2}$ Department of Hepatopancreatobiliary Surgery, The Second Affiliated Hospital of Guangzhou University of Chinese Medicine, Guangzhou, Guangdong, China

Full list of author information is available at the end of the article These authors contributed equally: Hang Sun, Shibo Sun, Gang Chen, Haorong Xie

Edited by A. Finazzi-Agrò

\section{Introduction}

Hepatectomy is frequently performed in the management of a variety of benign and malignant liver diseases. Post-hepatectomy liver dysfunction caused by impaired liver recovery is still a life-threatening morbidity ${ }^{1,2}$. Due to lack of efficient therapy, the mortality of posthepatectomy liver failure is reported at $50 \%{ }^{2}$. Clinical and experimental hepatectomy elicits a centric role of macrophages in regulating injury and regeneration in the liver ${ }^{3}$. Currently, the functional plasticity of macrophages is characterized by tissue-damaging or repairing properties ${ }^{4}$. Classical (M1) polarization driven by lipopolysaccharide (LPS) and alternative polarization (M2) driven

\section{(c) The Author(s) 2021}

(c) (i) Open Access This article is licensed under a Creative Commons Attribution 4.0 International License, which permits use, sharing, adaptation, distribution and reproduction cc. in any medium or format, as long as you give appropriate credit to the original author(s) and the source, provide a link to the Creative Commons license, and indicate if changes were made. The images or other third party material in this article are included in the article's Creative Commons license, unless indicated otherwise in a credit line to the material. If material is not included in the article's Creative Commons license and your intended use is not permitted by statutory regulation or exceeds the permitted use, you will need to obtain permission directly from the copyright holder. To view a copy of this license, visit http://creativecommons.org/licenses/by/4.0/. 
by interleukin-4 (IL-4) represent the extreme states associated with tissue injury and regeneration ${ }^{5}$. Elchaninov et al. reported that the increase of M2-like macrophages was likely correlated with liver regeneration after hepatectomy ${ }^{6}$. The study from Melgar-Lesmes et al. also demonstrated that the increased infiltration of M2 macrophages in the liver was linked to the enhancement of liver regeneration ${ }^{7}$. Notably, cytotherapy with polarized macrophages was found to alleviate certain hepatopathy by altering immune response in the liver, such as fibrosis ${ }^{8}$. These results altogether lead to a hypothesis that hepatoprotective macrophages which improve liver repair may have cytotherapeutic potential for post-hepatectomy liver dysfunction.

Emerging studies have demonstrated that bioactive lipids are critical in mediating the distinct functions of polarized macrophages in tissue damage and repair ${ }^{9-12}$. Giannakis et al. reported that dynamic changes in fatty acids support the phenotype transition of macrophages in muscle regeneration, providing substantial evidence for exploring lipid mediators in subtypes of macrophages which distinctly regulates tissue injury and regeneration ${ }^{9}$. Ceramides (CERs) and sphingosine-1-phosphate (S1P) in the sphingolipid metabolism are particularly highlighted to distinctly regulate hepatocyte injury and repair ${ }^{13}$. Accumulation of CER in hepatocytes has been reported to cause cell death and eventually lead to liver dysfunction, liver atrophy, and liver cancer ${ }^{14-16}$. In contrast to CER, S1P is a pro-survival lipid that is implicated in promoting tissue regeneration through enhancing cell proliferation and angiogenesis ${ }^{17,18}$. Lentsch and Hla's studies demonstrated that extracellular S1P is important in promoting liver regeneration by enhancing angiogenesis and hepatocyte proliferation ${ }^{19,20}$. However, much remains unknown about the bioactive lipids in mediating the functions of polarized macrophages in post-hepatectomy liver recovery.

In this study, we investigated the role of bioactive lipids that mediated the effects of polarized-macrophages infusion on post-hepatectomy liver recovery. Our study uncovers CER and S1P as critical lipids that mediate the hepatotoxic and hepatoprotective effects of M1- and M2macrophage infusion on liver recovery after hepatectomy, highlighting a cytotherapeutic capacity of M2 macrophage via generation of hepatoprotective S1P for posthepatectomy liver dysfunction.

\section{Materials and methods Mouse model}

C57BL/6 mice were bred and kept at the animal facility of Nanfang Hospital Southern Medical University on a $12 \mathrm{~h} / 12 \mathrm{~h} \mathrm{light} /$ dark cycle at $21^{\circ} \mathrm{C}$ and $50-55 \%$ humidity under specific pathogen-free (SPF) conditions. Mouse $2 / 3$ hepatectomy was performed as described by Mitchell and
Willenbring ${ }^{21}$. The liver regeneration index was demonstrated using the ratio of the remnant liver weight and body weight. Liver tissues for immunohistochemistry were fixed in $4 \%$ paraformaldehyde (PFA) (Biosharp, Hefei, China) then embedded in paraffin or Tissue-Tek OCT compound (Sakura Finetek, Chuo-ku, Tokyo, Japan). Liver tissues for RNA isolation, protein extraction, or sphingolipid extraction were snap-frozen in liquid nitrogen then stored at $-80^{\circ} \mathrm{C}$. Blood samples were allowed to clot at room temperature, and the serum was collected by centrifugution and then stored at $-80^{\circ} \mathrm{C}$ until analyzed. Serum total bilirubin (TBIL), alanine aminotransferase activity (ALT), and albumin (ALB) were determined using Bilirubin Assay Kit (Sigma, St. Louis, MO, USA), Alanine Transaminase Colorimetric Activity Assay Kit (Cayman Chemical, Ann Arbor, MI, USA), and Albumin Assay Kit (Sigma, St. Louis, MO, USA), respectively, following the manufacturer's instructions. All animal experiments were carried out following the protocols approved by the Institutional Animal Care and Use Committee of Nanfang Hospital Southern Medical University (NFYY-2017-03).

\section{Bone-marrow-derived macrophages culture, small interfering RNA transfection, and BMDM infusion}

Isolation, culture, and polarization of bone-marrowderived macrophages (BMDMs) were performed according to the protocol published by Ying et al. ${ }^{22}$. BMDMs were incubated with phosphate buffer saline (PBS) (Gibco, Waltham, MA, USA) as vehicle control, $100 \mathrm{ng} / \mathrm{ml}$ LPS (Sigma, St. Louis, MO, USA), and $10 \mathrm{ng} / \mathrm{ml} \mathrm{IL-4} \mathrm{(Pepro-}$ tech, Rocky Hill, NJ, USA) for $72 \mathrm{~h}$ to induce M1 and M2 polarization, respectively. For in vivo tracking after BMDM infusion, BMDM were transfected with green fluorescent protein (GFP) plasmid (Jikai, Shanghai, China) with Lipofectamine 2000 (Invitrogen, Waltham, MA, USA) before inducing polarization. For disturbing CER metabolism in polarized BMDMs, M1 and M2 BMDMs were transfected with small interfering RNA (siRNA) (Jikai, Shanghai, China) targeting Cers2 (5'-CCUACA CUGCACGAUGAUAUATT-3', 5'-UAUAUCAUCGUGC AGUGUAGGTT-3) or Sphk1 (5'-ACCUUCUUUCGCC UAGCAATT-3', 5'-UUGCUAGGCGAAAGAAGGUTT3) using X-treme GENE siRNA Transfection Reagent (Roche Diagnostics, Mannheim, Germany). Transfection was performed at $24 \mathrm{~h}$ before induction of polarization. Polarized BMDM infusion was performed as described ${ }^{8,23}$ with slight modification. Non-polarized (M0), M1, and M2 BMDMs were trypsinized from the 6-well plates and washed in $10 \mathrm{ml}$ of PBS three times by centrifugation (1000 r.p.m., $5 \mathrm{~min}$ ), then the collected BMDMs were resuspended in $100 \mu \mathrm{l}$ of PBS; $1 \times 10^{6}$ polarized BMDMs were delivered to the remnant liver via portal vein injection by a $33 \mathrm{G}$ microsyringe (Hamilton, Reno, NV, USA) 
after hepatectomy. Postoperative mice were sacrificed at designated time points and the tissues were processed as mentioned above.

\section{Untargeted lipidomics}

The conditional medium (CM) of polarized macrophages was collected at $48 \mathrm{~h}$ later after stimulation with LPS or IL-4, following by centrifuging at 1200 r.p.m. for 5 min to precipitate cell debris, and the supernatant was filtered by a $0.22-\mu \mathrm{m}$ syringe filter and lyophilized by a freeze dryer. The lyophilized medium was used for lipid extraction. Tissues and cells were homogenized on ice in buffer $[25 \mathrm{mM}$ tris (hydroxymethyl)-aminomethane hydrochloride (Tris-HCl), $\mathrm{pH} 7.4,150 \mathrm{mM} \mathrm{NaCl}, 1 \mathrm{mM}$ ethylene diamine tetraacetic acid (EDTA), and $1 \mathrm{mM}$ ethyleneglycoltetraacetic acid (EGTA)]. Lipid extraction was performed by using a modified Bligh and Dyer procedure as described previously ${ }^{24}$. Each individual lipid extract was reconstituted with a volume of $500 \mu \mathrm{l} /$ $\mathrm{mg}$ of protein or lyophilized powder of $2 \mathrm{ml}$ of the medium in $1: 1 \mathrm{CHCl}_{3} / \mathrm{MeOH}$, and then flushed with nitrogen. Quality control samples were established by pooling the examined cell or medium samples. The samples were analyzed by a Thermo Fisher Scientific Vanquish Flex ultra-high-performance liquid chromatography (UHPLC) equipped with Thermo Fisher Scientific Orbitrap Fusion Tribrid High-Resolution Mass Spectrometer (Thermo Fisher Scientific, Waltham, MA, USA). Briefly, $5 \mu$ l of the lipid extract was injected, and chromatographic separation was carried out at $40^{\circ} \mathrm{C}$ on an Acquity UPLC column C30 $(150 \times 2.1 \mathrm{~mm}, 3.0 \mu \mathrm{m})$. The mobile phase A was: $10 \mathrm{mM} \mathrm{HCOONH}_{4}, 0.1 \%$ formic acid, acetonitrile $(\mathrm{ACN}): \mathrm{H}_{2} \mathrm{O}=60: 40$, mobile phase $\mathrm{B}: 10 \mathrm{mM} \mathrm{HCOONH} \mathrm{H}_{4}, 0.1 \%$ formic acid, isopropanol (IPA): $\mathrm{ACN}=90: 10$. The electrospray ionization source was used with both positive and negative ion modes. Mass scan were performed as the following settings: orbitrap resolution: 60,000; scan range: $200-2000 \mathrm{~m} / z$; radiofrequency lens: $60 \%$; automatic gain control target: 2.0e5; maximum injection time: $100 \mathrm{~ms}$; polarity: positive. The identification of lipid molecular species was performed using Lipid Search software (Thermo Fisher Scientific; Waltham, MA, USA). These lipid-profiling data were then subjected to further analyses using the online Metaboanalyst tool (www.metaboanalyst.ca) ${ }^{25}$, the data first underwent a normalization using values of quality control samples, missing values for compounds that were not detected in all samples were excluded. $\log 2$ transformation was conducted to create a heatmap. Log transformation was performed, and the data were autoscaled for principal component analysis (PCA) and orthogonal projections to latent structures discriminant analysis (OPLS-DA) analyses.

\section{Targeted lipidomics}

The measurement of CER metabolites was performed according to the protocol reported by Wang et al. ${ }^{26}$. Tissues and cells were homogenized on ice in the buffer as aforementioned. Lipids from tissue homogenates ( $2 \mathrm{mg}$ protein per sample) were extracted with ethyl acetate/isopropanol/water (60/30/10, v/v/v). Lipids from the medium of polarized macrophages $(100 \mu \mathrm{l}$ diluted with $1900 \mu \mathrm{l}$ of serum-free RPMI medium) were extracted with ethyl acetate/isopropanol $(85 / 15, \mathrm{v} / \mathrm{v})$. The lipid extracts were dried under $\mathrm{N}_{2}$ gas stream and reconstituted in methanol, then CER, sphingosine (SPH), and S1P were determined by ultra-high-performance liquid chromatography-electrospray ionization mass spectrometry (UHPLC-ESI-MS/MS) performed on prelude SPLC + TSQ Quantiva LC-MS/MS system (Thermo Fisher Scientific, Waltham, MA, USA). Standards and internal standards of CER metabolites were purchased from Avanti Polar Lipids, Alabaster, AL, USA. Amounts of CER metabolites were quantified using standard curves of external standards, and then normalized to protein contents or medium volume.

\section{Immunohistochemistry}

Polarized macrophages in liver were immunofluorescent double-labeled using M.O.M. ${ }^{\circledR}$ (Mouse on Mouse) Basic Kit (Vector, Burlingame, CA, USA) according to the manufacturer's instructions. Anti-F4/80 (Abcam, Cambridge, MA, USA) was used to stain macrophages. Anti-inducible nitric oxide synthase (iNos) antibody (BD, San Jose, CA, USA) and anti-CD206 antibody (Bio-Rad, Hercules, CA, USA) were used to label M1 and M2 macrophages, respectively. Alexa Fluor $^{\circledR}$ 488conjugated antibody (Abcam, Cambridge, MA, USA), Alexa Fluor ${ }^{\circledR}$ 594-conjugated antibody (Abcam, Cambridge, MA, USA), and Cy3-conjugated antibody (Vector, Burlingame, CA, USA) were used as secondary antibodies. For the liver tissues with GFP-transfected macrophages infusion, anti-GFP antibody (Abcam, Cambridge, MA, USA) and Alexa Fluor ${ }^{\circledR}$ 488-conjugated secondary antibody were applied to detect GFP-positive macrophages. The stained sections and cells were mounted in an anti-fade solution with 4',6-diamidino-2-phenylindole (DAPI) (Abcam, Cambridge, MA, USA). Immunostaining on liver tissue sections was performed using VECTASTAIN $^{\circledR}$ Elite $^{\circledR}$ ABC HRP Kit (Vector, Burlingame, CA, USA) according to the manufacturer's instructions, antiproliferating cell nuclear antigen (PCNA) antibody (Cell Signaling Technology, Danvers, MA, USA) and anticleaved-caspase 3 (C-Caspase 3) antibody (Cell Signaling Technology, Danvers, MA, USA) were used to detect proliferation and apoptosis, respectively. Anti-hepatocyte nuclear factor $4 \alpha$ (HNF-4 $\alpha$ ) antibody (Abcam, Cambridge, MA, USA) was used to stain hepatocytes. Alexa Fluor ${ }^{\circledR}$ 
488-conjugated secondary antibody and Alexa Fluor ${ }^{\circledR}$ 568-conjugated secondary antibody (Abcam, Cambridge, MA, USA) were used as secondary antibodies. Terminaldeoxynucleoitidyl transferase-mediated nick end labeling (TUNEL) assay on cells or liver sections was performed using a TdT In Situ Apoptosis Detection Kit (Minneapolis, MN, USA) according to manufacturer's instructions. Stained cells and liver sections were observed, and positive cells were counted in five random fields under Intelligently Designed Microscope (Olympus, Shinjukuku, Tokyo, Japan) or LSM 880 with Airyscan Confocal Microscope (Zeiss, Oberkochen, Germany).

\section{Western blotting}

Liver or cell homogenates were prepared in radioimmune precipitation buffer (RIPA) $[50 \mathrm{mmol} / \mathrm{L}$ tris (hydroxymethyl)-aminomethane (Tris), 1\% NP40, 0.25\% deoxycholic acid sodium salt, $150 \mathrm{mmol} / \mathrm{L} \mathrm{NaCl}, 1 \mathrm{mmol} /$ L EGTA] containing $1 \mathrm{mM}$ phenylmethanesulfonyl fluoride (PMSF) and a protease inhibitor cocktail (Roche, Indianapolis, IN, USA). Protein concentrations were measured with a BCA Protein Assay Kit (Invitrogen, Waltham, MA, USA) according to manufacturer's manual. Thirty micrograms of protein extracts were denatured in Laemmli buffer containing $5 \% \beta$-mercaptoethanol, then loaded and separated by gel electrophoresis on a $10 \%$ or $12 \%$ Bis-Tris gel. Primary antibodies were incubated at $4{ }^{\circ} \mathrm{C}$ overnight under shaking conditions. Immunoreactive bands were visualized on nitrocellulose filter membrane using horseradish peroxidase (HRP)-linked anti-mouse or anti-rabbit antibody (Cell Signaling Technology, Danvers, MA, USA) and the ECL Ultra Western HRP Substrate (Millipore, Burlington, MA, USA). Anti-PCNA antibody (Cell Signaling Technology, Danvers, MA, USA), anti-CCaspase 3 antibody (Cell Signaling Technology, Danvers, MA, USA), and anti-cleaved PARP (C-PARP) antibody (Cell Signaling Technology, Danvers, MA, USA) were used to evaluate cell proliferation and apoptosis. The expression level of $\beta$-Actin (Abcam, Cambridge, MA, USA) was used as an internal control. Antibodies used in this study are listed in Supplemental Table 1.

\section{Quantitative reverse transcription PCR}

Total RNA was extracted from cells or liver tissues using PureLink ${ }^{\mathrm{TM}}$ RNA Mini Kit (Invitrogen, Waltham, MA, USA) according to the manufacturer's instructions. mRNA was reversely transcribed to cDNA by PrimeScript $^{\mathrm{TM}}$ RT Master Mix (TaKaRa, Kusatsu, Shiga, Japan). Quantitative reverse transcription PCR (qPCR) was performed to determine the mRNA levels of M1macrophage-associated genes, Inos, tumor necrosis factor- $\alpha(\operatorname{Tnf}-\alpha)$, and interleukin-6 (IL-6), M2-macrophageassociated genes, arginase1 (Arg1), inflammatory zone 1 (Fizz1), Il-4, and interleukin-10 (Il-10), and genes encoding CER metabolic enzymes. cDNA templates were diluted 1:5 and amplified with SYBR ${ }^{\circledR}$ Premix Ex Taq ${ }^{\mathrm{TM}}$ II (TaKaRa, Kusatsu, Shiga, Japan) using Roche LightCycler $^{\circledR} 480$ PCR System (Roche, Indianapolis, IN, USA). An initial denaturation at $95^{\circ} \mathrm{C}$ for $3 \mathrm{~min}$ was followed with PCR cycling: $95^{\circ} \mathrm{C}(5 \mathrm{~s})$ and $60^{\circ} \mathrm{C}(30 \mathrm{~s})$ for 40 cycles. Relative mRNA levels were calculated by means of $2^{-\Delta \Delta \mathrm{Ct}}$. $\beta$-Actin was used as the reference gene. Primer sequences used to amplify specific gene fragments are shown in Supplemental Table 2.

\section{Primary mouse hepatocytes and hepatic non-parenchymal cells isolation}

Primary mouse hepatocytes were harvested from postoperative mice by a modified rapid two-step perfusion $\operatorname{method}^{27}$. Briefly, $48 \mathrm{~h}$ after hepatectomy, mice were retrogradely perfused from the inferior vena cava with Hank's balanced salt solution (HBSS) and Dulbecco's modified eagle medium (DMEM) containing collagenase IV $(80 \mathrm{U} / \mathrm{ml})$ (Worthington Industries, Columbus, $\mathrm{OH}$, US) to digest liver tissues, and primary mouse hepatocytes were harvested from the digested liver by filtration through a 200-mesh filter followed by centrifugation at $500 \times g$ for $2 \mathrm{~min}$ for three times. The remaining cells were resuspended in 30\% Percoll followed by centrifugation at $450 \times g$ for $20 \mathrm{~min}$ to remove cell debris; $25 \%$ and $50 \%$ stock isotonic Percoll (SIP) were prepared by mixing Percoll solution (GE Healthcare Life Sciences, Marlborough, MA, US) with PBS (Gibco, Waltham, MA, USA). A centrifuge tube containing $50 \%$ SIP solution $(20 \mathrm{ml})$ and $25 \%$ SIP solution $(20 \mathrm{ml})$ was prepared. Then the hepatic non-parenchymal cells (HNPCs) were added to the SIP of discontinuous isotonic gradient and centrifuged at $800 \times g$ for $15 \mathrm{~min}$. The HNPCs, including hepatic macrophages, were collected from the $25 \%$ SIP cushion ${ }^{28}$. After lysis of red blood cells, the HNPCs were washed and resuspended for fluorescence-activated cell sorting (FACS) analyses. Primary mouse hepatocytes were resuspended and cultured in DMEM for $24 \mathrm{~h}$ in 6-well plates and confocal dishes, then the medium was changed with $2 \mathrm{ml} \mathrm{CM}$ of polarized macrophages, or the medium was supplemented with CER metabolites, including $10 \mu \mathrm{M}$ d18:1/ $\mathrm{C}_{16}$-CER (Avanti Polar Lipids, Alabaster, AL, USA) and $500 \mathrm{nM}$ d18:1-S1P (Avanti Polar Lipids, Alabaster, AL, USA). After 48-h stimulation, hepatocytes were collected for immunostaining, protein extraction, and RNA isolation, as aforementioned.

\section{3-(4,5-Dimethylthiazol-2-yl)-2,5 diphenyl tetrazolium assay} 3-(4,5-Dimethylthiazol-2-yl)-2,5 diphenyl tetrazolium (MTT, Merck-Calbiochem, Burlington, MA, USA) was dissolved in Milli $Q$ water $(5 \mathrm{mg} / \mathrm{ml})$. Primary mouse hepatocytes cultured in 12-well plates were incubated with a $300-\mu l$ mixture of MTT reagent $(5 \mathrm{mg} / \mathrm{ml})$ for $2 \mathrm{~h}$. 


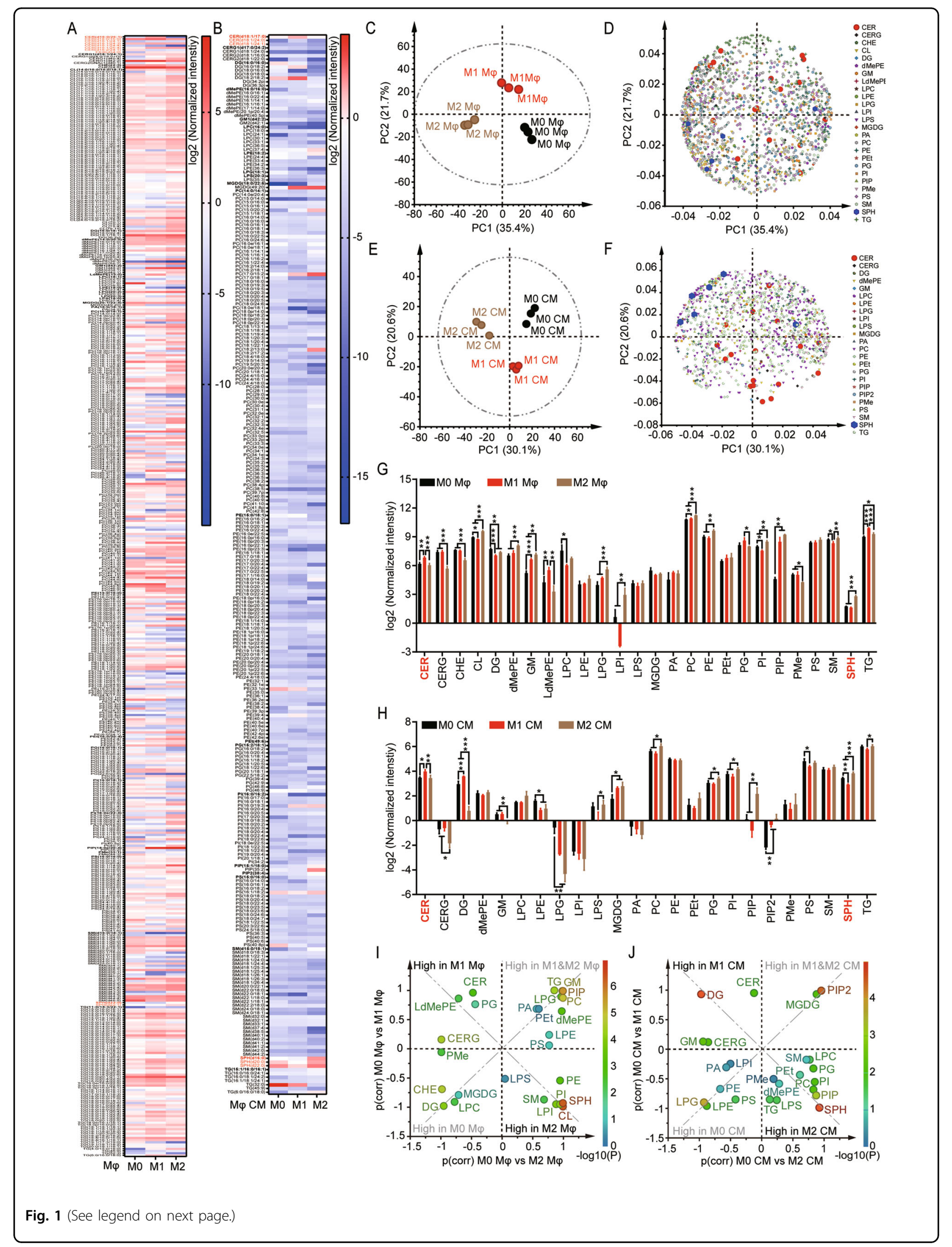


(see figure on previous page)

Fig. 1 Untargeted lipidomics on polarized BMDMs. Mouse bone marrow cells were differentiated and polarized into M1 and M2 BMDMs by treatment of LPS and IL-4, respectively. Lipids were extracted from non-polarized and polarized BMDMs and their CM, then subjected to untargeted lipidomics analyses by UHPLC-ESI-MS/MS. A, B Content of individual lipid species in M0 or polarized BMDMs (A) and their CM (B). CER and SPH species were highlighted in red. C-F Lipid profiles of MO, M1, M2 BMDM cells and their CM were detected by untargeted lipidomics. PCA score plots and loading plots were used for exploratory data analysis of lipid dysregulation in BMDMs (C and $\mathbf{D})$ and their CM (E and $\mathbf{F})$ through dimensional reduction and data visualization. G, H Content of lipid classes in M0, M1, and M2 BMDMs (C) and their CM (D). I, J SUS-plot analysis based on orthogonal projections to OPLS-DA on lipid classes in polarized BMDMs (I) and their CM (J). Data represent results from three samples, each was pooled using BMDMs isolated from three mice. Data in $\mathbf{G}$ and $\mathbf{H}$ represent mean $\pm \mathrm{SD}$; ${ }^{*} p<0.05,{ }^{* *} p<0.01$, ${ }^{* *} p<0.001$.

Then, $300 \mu \mathrm{l}$ of lysis buffer $(1 \mathrm{mM} \mathrm{HCL}$ and $10 \%$ Triton $\mathrm{X}-100$ in isopropanol) was added, plates were incubated at room temperature and gently shaken at 70 r.p.m. for 30 min to lysate the cells and elute MTT dye. The absorbance was then measured at $590 \mathrm{~nm}$ used a spectrometer.

\section{FACS analysis}

BMDMs or BMDMs with GFP-transfected were stained with PE-labeled anti-F4/80 (eBioscience, San Diego, CA, USA) or FITC-labeled anti-CD11b (eBioscience, San Diego, CA, USA). Prepared HNPCs were resuspended in PBS at $4{ }^{\circ} \mathrm{C}$ and pre-incubated with TruStain $\mathrm{FcX}^{\mathrm{TM}}$ (antimouse CD16/32) antibody (BioLegend Way, San Diego, CA, USA) to minimize non-specific antibody binding, then HNPCs were stained with PE-labeled anti-F4/80 antibody (eBioscience, San Diego, CA, US), FITC-labeled anti-CD11b antibody (eBioscience, San Diego, CA, US), and anti-GFP antibody (Abcam, Cambridge, MA, USA) with secondary antibody Alexa Fluor ${ }^{\circledR}$ 647-conjugated antibody (Abcam, Cambridge, MA, USA). FACS analysis was performed using a FACS Calibur ${ }^{\mathrm{TM}}$ flow cytometer (BD Immunocytometry Systems, San Jose, CA, USA). Data were analyzed with the FlowJo software version 10.0 (FlowJo, Ashland, OR, USA).

\section{Statistics}

Data are expressed as the mean \pm standard deviation (SD). Statistical analyses were performed using one-way analysis of variance (ANOVA) followed by Tukey's posthoc test or a Student's $t$-test. $P<0.05$ was considered statistically significant. Data analysis was performed using IBM SPSS Statistics for Windows version 22.0 (IBM; Armonk, NY, USA).

\section{Results}

Lipidomics reveals elevation of CER metabolites as signature lipid species relevant to M1/M2 polarization in BMDM

Bioactive lipids produced by polarized macrophages have been implicated in mediating the distinct functions of polarized macrophages on tissue damage and repair ${ }^{29}$. In order to study lipids that have the potential to mediate the regulatory effects of polarized macrophages on liver injury and regeneration after hepatectomy, we first performed untargeted lipidomics analysis on polarized macrophages and their CM. Mouse bone marrow cells were isolated and differentiated into F4/80-positive BMDM cells (Supplementary Fig. $1 \mathrm{~A}-\mathrm{C}$ ), then polarized to M1 BMDMs or M2 BMDMs by LPS or IL-4 stimulation, respectively (Supplementary Fig. 1D). Overall, a total of 1659 and 1251 individual lipid species were determined in the polarized macrophages and their $\mathrm{CM}$, including lipid classes of CER, glycosyceramides (CERG), cholesterol ester (CHE), cardiolipins (CL), diglycerides (DG), dimethyiphosphatidylethanoiamines (dMePE), ganglioside (GM), lysodimethyiphosphatidylethanoiaminse (LdMePE), lysophosphatidylcholines (LPC), lysophosphatidylethanolamines (LPE), lysophosphatidylglycerols (LPG), lysophosphatidylinositols (LPI), lysophosphatidylserines (LPS), monogalactosyldiacylglycerol (MGDG), phosphatidic acid (PA), phosphatidylcholines (PC), phosphatidylethanolamines (PE), phosphatidylethanols (PEt), phosphatidylglycerols (PG), phosphatidylinositols (PI), phosphatidylinositol biphosphate $\left(\mathrm{PIP}_{2}\right)$, phosphatidylinositol phosphate (PIP), phosphatidylmethanol (PMe), phosphatidylserines (PS), sphingomyelins (SM), SPH, and triglycerides (TG). In total, 514 and 281 individual lipid species were significantly altered in response to polarization in macrophages and their medium, respectively (Fig. 1A, B). PCA demonstrated the contents of individual lipid species in macrophages (Fig. 1C, D), and their CM (Fig. 1E, F) were clearly separated as classification of M1, M2, and M0. Notably, the levels of most detected CER species were found to be high in M1 macrophages (Fig. 1A, C, D). Whereas the CER-derived lipid metabolites, SPH, were high in M2 macrophages (Fig. 1B, E, F). To further highlight the distinct variations of lipid classes in polarized macrophages and their CM, we performed OPLS-DA (Supplementary Fig. 2) and shared and unique structure plot (SUS-plot) analysis on the content of lipid classes (Fig. 1C-F). The results revealed that the levels of CER, CERG, LdMePE, PG, DG, and GM were significantly upregulated in response to $\mathrm{M} 1$ polarization (Fig. 1G-J), among which, CERs were the most significantly increased lipid class in both M1 macrophages and their CM (Fig. 1I, J and Supplementary Fig. 2). On the other hand, among LPS, SM, PI, PE, and SPH that were significantly elevated in response to $\mathrm{M} 2$ polarization (Fig. 1G-J), the levels of CER-derived metabolites, SPH, were uncovered as the 
most significantly elevated lipid class in both M2 macrophages and their CM (Fig. 1I, J and Supplementary Fig. 2). These data identified CERs and their metabolites SPH as critical lipid signatures in differentiating M1 and M2 polarization in macrophages.

\section{M1 and M2 BMDMs highly express CER-synthetic and CER-} catabolic pathways to produce CER and S1P, respectively

As we found that the levels of CER and their metabolites $\mathrm{SPH}$ were distinctly regulated in M1 and M2 BMDMs, we examined the difference in CER metabolic pathways in the polarized BMDMs by measuring the mRNA levels of genes encoding CER-metabolizing enzymes. We found that M1 macrophages expressed a CER-generationmetabolic pattern, which was characterized by upregulation of ceramide synthases (CERS) and hydrolase of SM and glycosphingolipids (GSL) that contribute to CER production, including Cers2, Cers3, Cers6, neutral sphingomyelinase 1 (Smpd2), neutral sphingomyelinase 2 (Smpd3), acid sphingomyelinase like 3A (Smpdl3a), and galactosylceramidase (Galc), and by downregulation of ceramidases (CDase) and sphingosine kinase (SPHK) which are involved in CER catabolism, including alkaline ceramidase 2 (Acer2), alkaline ceramidase 3 (Acer 3 ), neutral ceramidase (Asah2), and Sphk1 (Fig. 2A). However, M2 macrophages expressed a CER-catabolic pattern, which was characterized by dramatic upregulation of CDase and SPHK that hydrolyze CER, and synthase of SM and GSL that consume CER as substrates, including Acer2, Acer3, acid ceramidase (Asah1), Asah2, Sphk1, sphingomyelin synthase 1 (Sgms1), sphingomyelin synthase 2 (Sgms2), and UDP-glucose ceramide glucosyltransferase $(U g c g)$ (Fig. 2A). S1P Lyase (Sgpl) that encoded the S1P hydrolase was downregulated in both M1 and M2 macrophages, S1P phosphatase 1 (Sgpp1) that catalyzes the dephosphorylation of S1P was downregulated in M1 macrophages (Fig. 2A).

Catabolism of CER is known to produce $\mathrm{SPH}$, and $\mathrm{SPH}$ is then phosphorylated by SPHK to generate S1P. More importantly, S1P is currently found as a key signaling lipid that promotes hepatocyte proliferation and survival ${ }^{19,20,30}$. Since we found the mRNA levels of CDase and SPHK were dramatically upregulated by M2 polarization. We determined if S1P levels were consequently increased by M2 polarization. Therefore, we performed targeted lipidomics on d18:1-CER species, d18:1-SPH, and d18:1-S1P in polarized BMDMs and their CM. In accordance with the changes in metabolic enzymes, the levels of most d18:1-CER species were found to be significantly increased by $\mathrm{M} 1$ polarization (Fig. 2B-F), while the levels of d18-SPH and d18:1-S1P were significantly elevated in response to $\mathrm{M} 2$ polarization (Fig. 2B-F). These data collectively demonstrated that M1 macrophages expressed a CER-synthesis-dominant metabolism leading to an increase in CER accumulation, while M2 macrophages expressed a CER-catabolic metabolism resulting in upregulation of CER-derived S1P production.

\section{Infusion of M1 or M2 BMDMs correspondingly alters macrophage polarization and promotes CER or S1P generation in mouse liver after partial hepatectomy}

Infusion of polarized macrophages has been applied to study the pathophysiological functions and cytotherapeutic potential of macrophage polarization ${ }^{8}$. To further investigate the functions of polarized macrophages in regulating hepatic CER metabolism and liver recovery after hepatectomy, artificially polarized BMDMs were infused into the remnant liver tissues after hepatectomy. The mouse model for studying post-hepatectomy liver injury and regeneration was established by $2 / 3$ hepatectomy (Supplementary Fig. 3). A group of mice were first infused with BMDMs transfected with GFP, and both fluorescent microscopy and FACS analyses demonstrated that the GFP-transfected BMDMs indeed infiltrated into liver tissues after surgery (Supplementary Fig. 4). The polarized BMDMs were then infused into the remnant liver tissues of the donor's littermate mice after hepatectomy. Confocal scanning validated that the exogenous M1 and M2 BMDMs indeed infiltrated into mouse liver and survived during liver recovery after hepatectomy (Fig. 3A). To investigate if the infusion of polarized BMDMs altered macrophage polarization in liver tissues, we examined the change in portions of polarized macrophages in the remnant liver after hepatectomy. We found that M1-BMDM and M2-BMDM injection correspondingly increased the portion of iNos-positive M1 macrophages and CD206-positive M2 macrophages (Fig. 3B-D). Simultaneously, M1-BMDM infusion was found to upregulate M1associated genes in the remnant liver, including Tnf- $\alpha, I l-6$, and $i N o s$ (Fig. 3E), whereas M2-BMDM infusion was found to elevate the expression of M2-associated genes in the remnant liver, including Fizz1, Arg1, Il-4, and Il-10 (Fig. 3F).

Since M1 BMDMs and M2 BMDMs preferred producing CER and S1P, respectively, targeted lipidomics on d18:1-CER, d18:1-SPH, and d18:1-S1P were further performed to examine whether polarized BMDM infusion correspondingly increased the levels of CER and S1P in the remnant liver after hepatectomy. Indeed, we found that M1-BMDM infusion significantly increased the levels of most d18:1-CER species without affecting d18:1-SPH and d18:1-S1P (Fig. 3G, H). However, M2-BMDM infusion significantly reduced the levels of most d18:1-CER species and elevated the level of d18:1-SPH and d18:1-S1P in the remnant liver tissues (Fig. 3G, H). These data altogether demonstrated that infusion of M1 macrophages enhanced M1 polarization and promoted CER accumulation, while M2 macrophages infusion enhanced M2 polarization and promoting S1P production in the remnant liver after hepatectomy. 


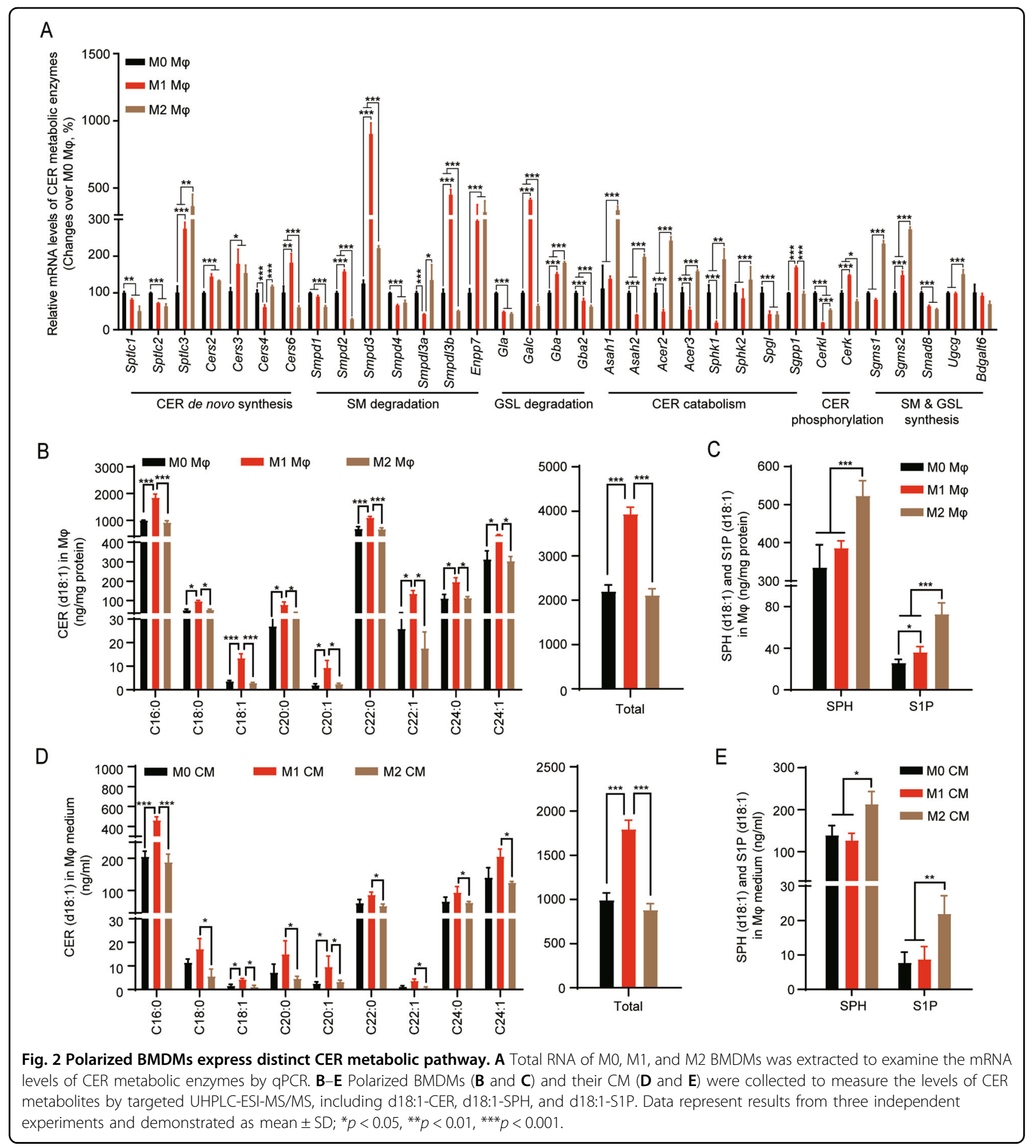

Infusion of M1 and M2 BMDMs exerts distinct effects on promoting either injury or regeneration in mouse liver after partial hepatectomy

Since we found that the infusion of polarized BMDMs altered macrophage polarization and CER metabolites in mouse liver after hepatectomy, we further examined if the infusion of artificially polarized BMDMs affected liver injury and regeneration after hepatectomy. Liver regeneration index measurement revealed that M1-BMDM injection inhibited liver regeneration, while M2-BMDM infusion promoted liver regeneration (Fig. 4A). Morphological examination on the remnant livers demonstrated that M1-BMDM-infused mice had significantly smaller liver, while M2-BMDM-infused mice had larger liver 


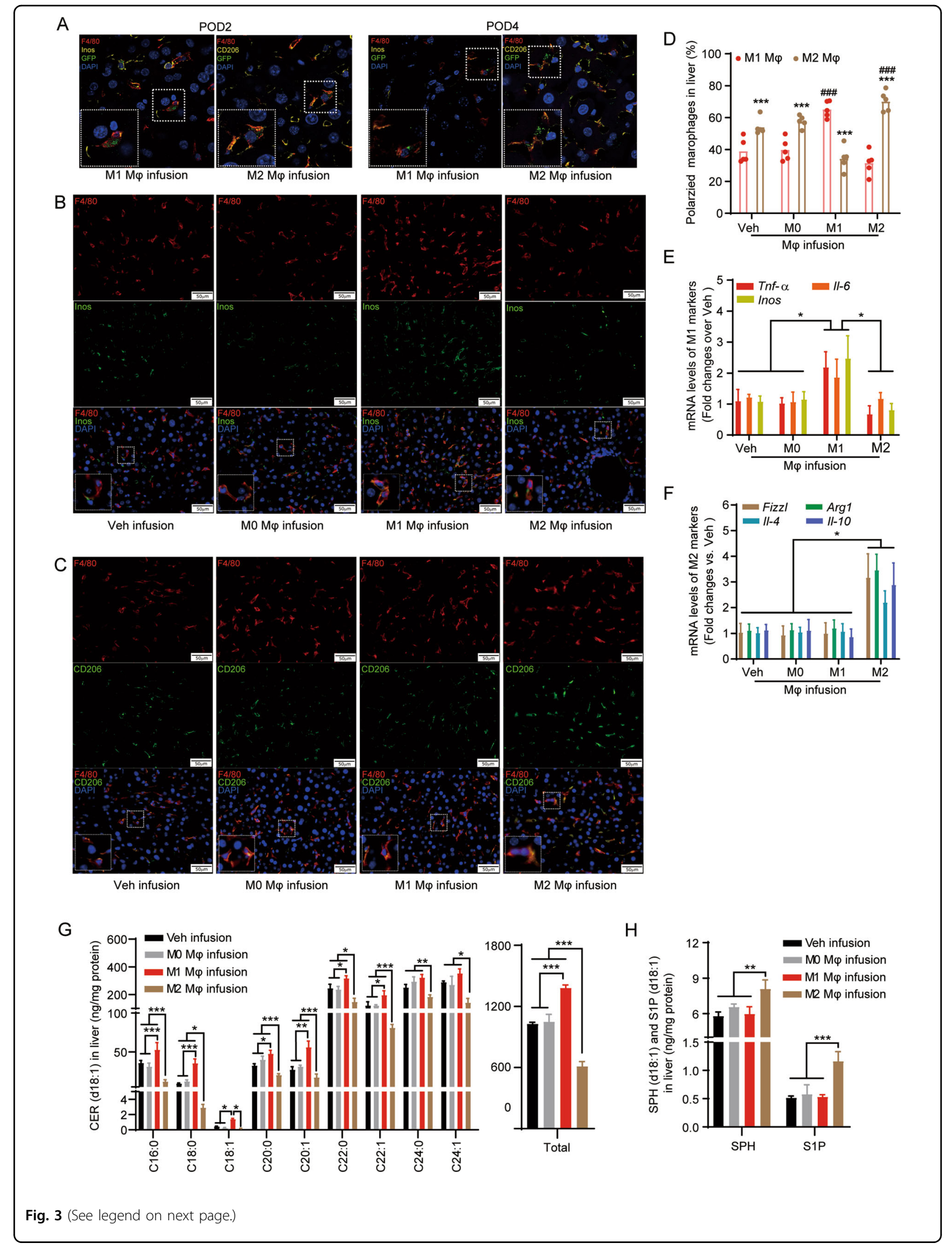


(see figure on previous page)

Fig. 3 Infusion of ex vivo polarized BMDMs alters macrophage polarization and CER metabolism in the liver after partial hepatectomy. Mouse livers after hepatectomy were infused with phosphate buffer saline (PBS) (Veh), M0, M1, and M2 BMDMs. The remnant liver tissues were collected at postoperative day 2 (POD2) to examine macrophage polarization and levels of CER metabolites. A Liver tissues were harvested at 2 days and 4 days after GFP-positive polarized BMDM infusion. Liver sections were stained with anti-GFP (green), anti-F4/80 (macrophage marker, red), antiInos (M1 marker, yellow), and anti-CD206 antibodies (M2 marker, yellow) to validate the infiltration of polarized BMDM in the remnant liver after hepatectomy by confocal scanning. DAPI was used to visualize nuclei (blue). B, C Liver sections were co-stained with F4/80 (red) and iNos (green) (B) or CD206 (green) (C) to examine M1 or M2 macrophage polarization in response to BMDM infusion in the remnant liver after hepatectomy. DAPI was used to visualize nuclei (blue). D M1 and M2 macrophages in the remnant liver tissues were counted in five random 20x fields to quantify alteration in $\mathrm{M} 1$ and $\mathrm{M} 2$ polarization by BMDM infusion in the remnant liver after hepatectomy. $\mathbf{E}, \mathbf{F}$ Total RNA was extracted from the remnant liver tissues, then mRNA levels of M1-macrophage-associated genes (Tnf-a, Inos, and II-6) (E) and M2-macrophage-associated genes (Fizzl, Arg1, II-4, and II-10) (F) were determined by qPCR. G, $\mathbf{H}$ Levels of CER metabolites were determined in the remnant liver tissues with BMDM infusion after hepatectomy, including d18:1-CER (G), d18:1-SPH (H), and d18:1- S1P $(\mathbf{H})$ by targeted UHPLC-ESI-MS/MS. Images in $\mathbf{A}-\mathbf{C}$ represent results from five individual mice in each group. Data in $\mathbf{E}-\mathbf{H}$ are demonstrated as mean $\pm \mathrm{SD}, n=5-6 ;{ }^{*} p<0.05,{ }^{* *} p<0.01,{ }^{* * *} p<0.001$.

(Fig. 4B). To examine the effects of BMDM infusion on liver regeneration and liver injury, the expression levels of proliferation marker and apoptotic maker in the liver tissues were measured. Immunoblotting demonstrated that M2-BMDM infusion elevated the proliferation marker PCNA and M1-BMDM infusion upregulated the apoptosis marker C-PARP (Fig. 4C). To further examine the hepatocyte-specific effects of BMDM infusion, HNF- $4 \alpha$ antibody was used to label hepatocytes, then C-Caspase 3 and PCNA antibody were used to label apoptotic and proliferative cells, respectively. Immunofluorescent staining revealed that M2-BMDM infusion significantly increased the portion of PCNA/HNF- $4 \alpha$ double-positive hepatocytes and the M1-BMDM infusion significantly increased the portion of $\mathrm{C}$-caspase $3 / \mathrm{HNF}-4 \alpha$ doublepositive hepatocytes (Fig. 4D-F), indicating that the M2BMDM infusion indeed promoted hepatocellular proliferation and the M1-BMDM infusion worsened hepatocellular apoptosis in the hepatectomized liver. Liver function test further revealed that M1-BMDM infusion significantly elevated the levels of ALT and TBIL and reduced the levels of ALB, indicating M1-BMDM infusion aggravated post-hepatectomy liver dysfunction (Fig. 4H-J). M2-BMDM infusion significantly decreased the levels of ALT and ALB, but had no significant effect on TBIL levels (Fig. 4H-J). These data suggested that M1-BMDM infusion worsened hepatocyte apoptosis and aggravated liver dysfunction after hepatectomy, while M2-BMDM infusion promoted hepatocyte proliferation and attenuated posthepatectomy liver dysfunction.

\section{CER and S1P mediate the functions of M1 and M2 BMDMs on regulating post-hepatectomy hepatocyte apoptosis and proliferation}

Emerging studies implicate CER and S1P as essential bioactive lipids in regulating tissue injury and regeneration. In order to test if CER and S1P indeed mediate the distinct effects of BMDM infusion in promoting hepatocyte death and proliferation, we isolated mouse primary hepatocytes from the remnant liver after hepatectomy and then incubated them with the CM collected from BMDM or exogenous CER and S1P. MTT assay demonstrated that the viability of hepatocytes treated with the CM from M1 BMDMs was significantly reduced (Fig. 5A). However, hepatocyte viability was significantly increased by the treatment of M2-BMDM CM (Fig. 5A). The immunostaining results of apoptosis marker CCaspase 3 and proliferation marker PCNA further revealed that M1-BMDM CM increased the number of C-Caspase 3 positive hepatocytes (Fig. 5B, C), but M2$\mathrm{BMDM} C \mathrm{CM}$ elevated the number of PCNA-positive hepatocytes (Fig. 5B, D). These data indicate that M1BMDM CM augments apoptosis, but M2-BMDM CM promotes proliferation in post-hepatectomy hepatocytes. Therefore, secretion from polarized macrophages indeed contributes to regulating proliferation and apoptosis in post-hepatectomy hepatocytes.

Next, we measured the levels of d18:1-CER, d18:1-SPH, and d18:1-S1P in primary hepatocytes incubated with the CM from polarized BMDMs. We found that M1-BMDM medium treatment significantly increased the levels of most d18:1-CER species in post-hepatectomy primary hepatocytes (Fig. 5E, F). However, treatment with M2BMDM medium reduced the levels of most d18:1-CER species and elevated the levels of d18:1-SPH and d18:1S1P in post-hepatectomy primary hepatocytes (Fig. 5E, F). To further evaluate the effects of CER and S1P on hepatocyte viability after hepatectomy, post-hepatectomy primary hepatocytes were treated with exogenous d18:1/ $\mathrm{C}_{16: 0^{-}}$CER and d18:1-S1P. Examination of cell viability showed that $\mathrm{d} 18: 1 / \mathrm{C}_{16: 0}$-CER reduced cell viability, whereas d18:1-S1P increased cell viability (Fig. 5G). Immunoblotting consistently demonstrated that $\mathrm{d} 18: 1 /$ C16:0-CER treatment upregulated the protein levels of apoptosis marker C-Caspase 3 (Fig. 5H). However, d18:1S1P increased the protein levels of proliferation marker PCNA in post-hepatectomy hepatocytes (Fig. 5H), suggesting that $\mathrm{d} 18: 1 / \mathrm{C} 16: 0-\mathrm{CER}$ induced apoptosis, but d18:1-S1P promoted proliferation in post-hepatectomy hepatocytes. 


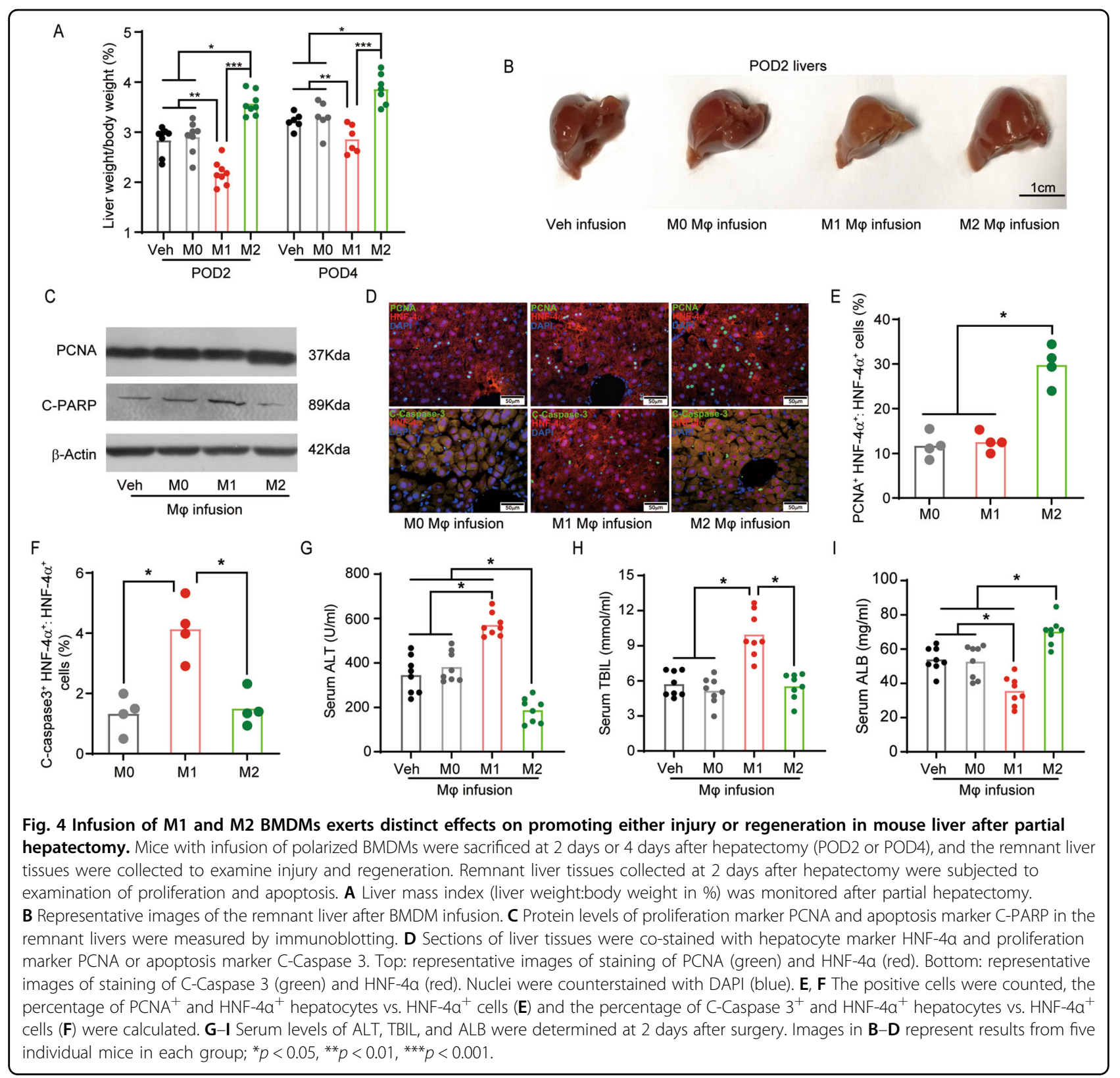

To further verify that d18:1-CER and d18:1-S1P distinctly mediated the pro-death and pro-recovery effects of infused M1 and M2 BMDMs in post-hepatectomy liver. We knocked down Cers2 and Sphk1 in M1 and M2 BMDMs by siRNA transfection, respectively (Fig. 6A, B). Cers2 catalyzes the synthesis of long-chain $\mathrm{CER}^{31,32}$, and Cers2 was found to be upregulated in the M1 BMDMs (Fig. 2A). Sphk1 catalyzes the phosphorylation of SPH to produce $S 1 \mathrm{P}^{33-35}$, and Sphk1 was found to be upregulated in the M2 BMDMs (Fig. 2A). Consistently, targeted lipidomics demonstrated that CerS2 knockdown decreased the levels of long-chain CER species and total CER in M1 BMDMs and their CM, Sphk1 knockdown decreased the levels of S1P and accumulated SPH in M2 BMDMs and their $\mathrm{CM}$ (Fig. 6C-F). The post-hepatectomy primary hepatocytes were treated with the CM from these transfected BMDMs, and the proliferation and apoptosis of the hepatocytes were examined. Immunostaining revealed that Cers2 knockdown attenuated the pro-apoptotic effects of M1 CM, but Sphk1 knockdown endowed the M2 CM with pro-apoptotic effects instead of attenuating its pro-survival effects (Fig. 6G-I). Next, MTT assay showed that Cers2 knockdown attenuated the M1CMinduced decreased of cell viability (Fig. 6J). Notably, CM of Sphk1-knockdown M2 BMDMs reduced cell viability in hepatocytes (Fig. 6J). 


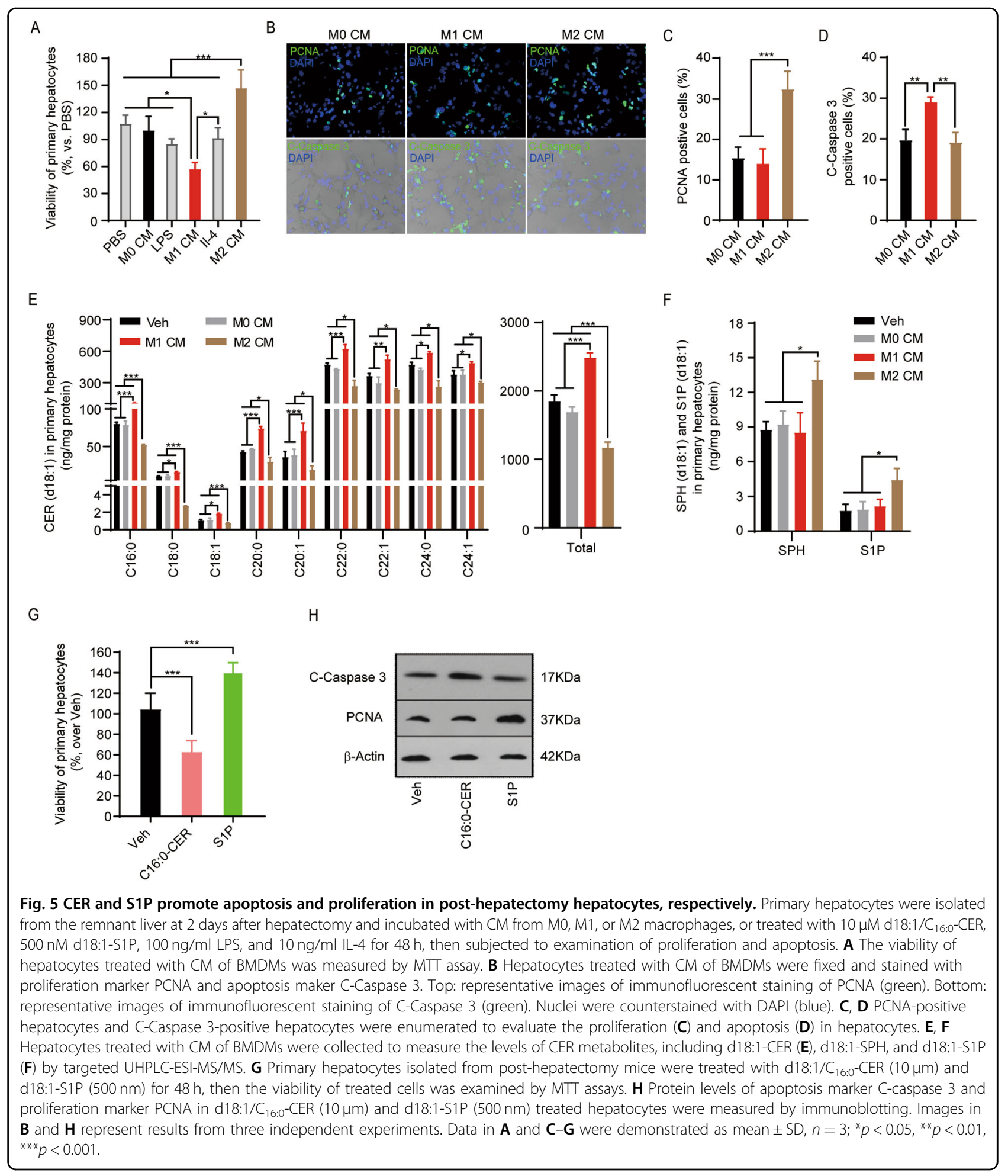

These data collectively demonstrated that CER and S1P were critical lipids that mediated the effects of M1- and M2-BMDM infusion on promoting apoptosis and proliferation in post-hepatectomy hepatocytes, respectively.

\section{Discussion}

Post-hepatectomy liver dysfunction caused by impaired liver recovery is a life-threatening morbidity, yet without efficient therapy ${ }^{36}$. Aiming to investigate the therapeutic capacity of polarized macrophages and the relevant lipid 


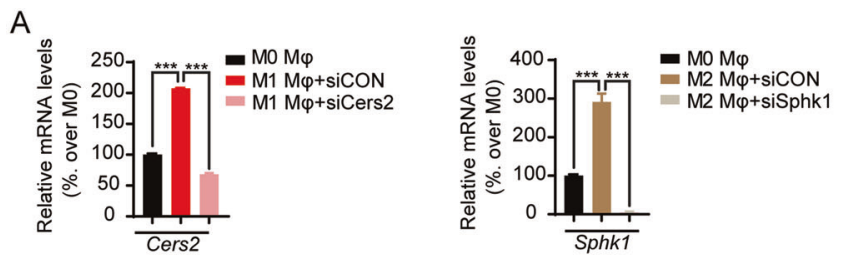

C
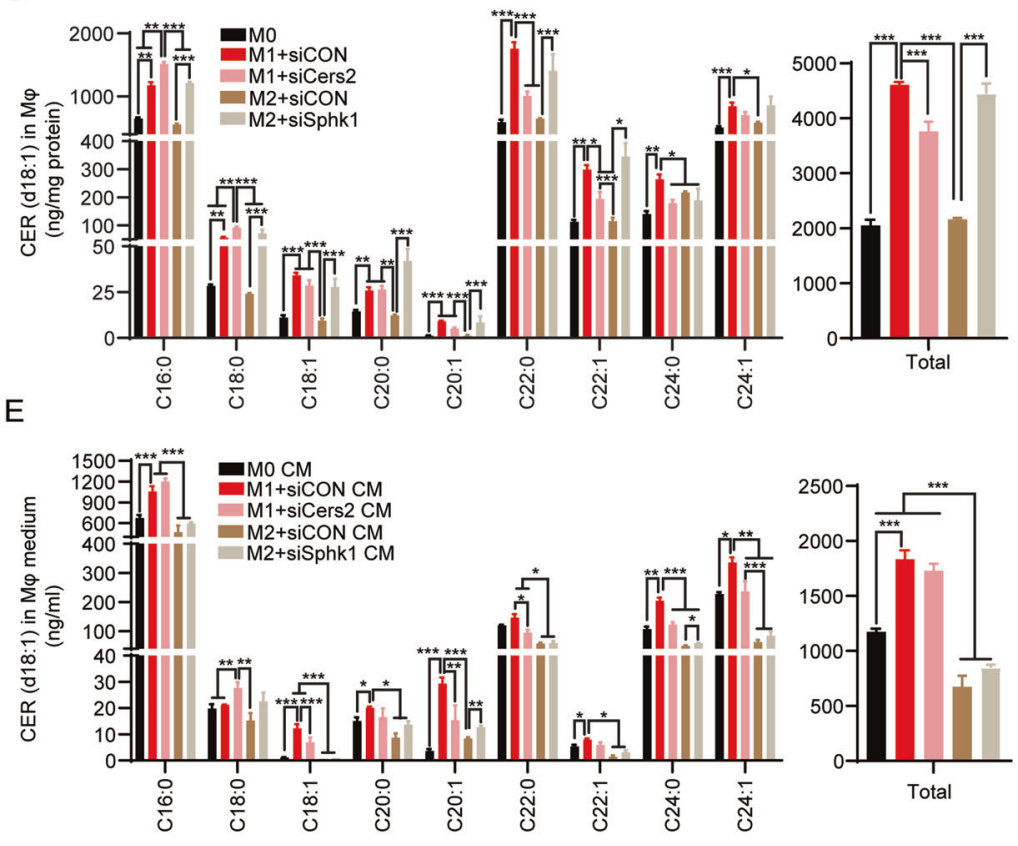

B

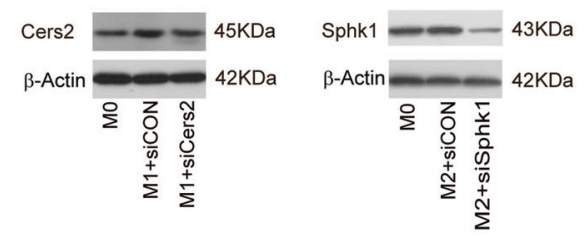

D

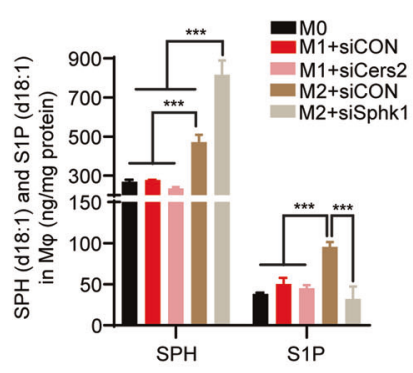

$\mathrm{F}$

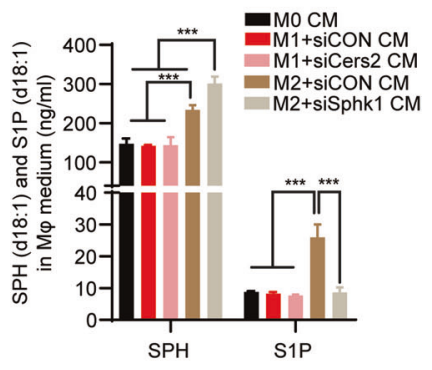

G

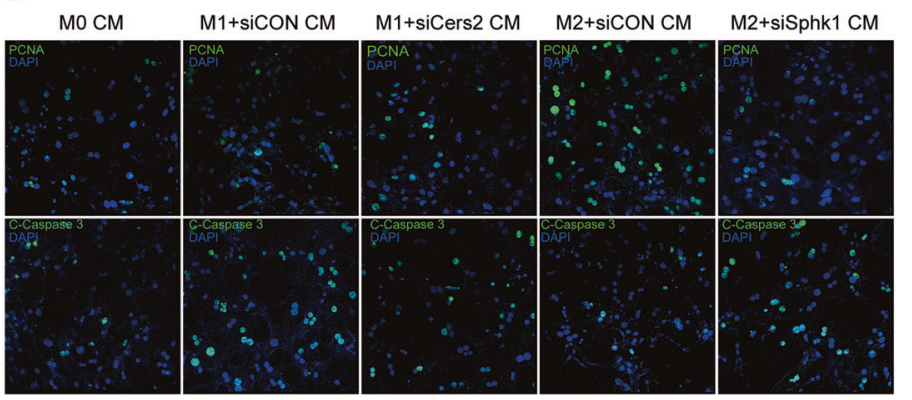

H
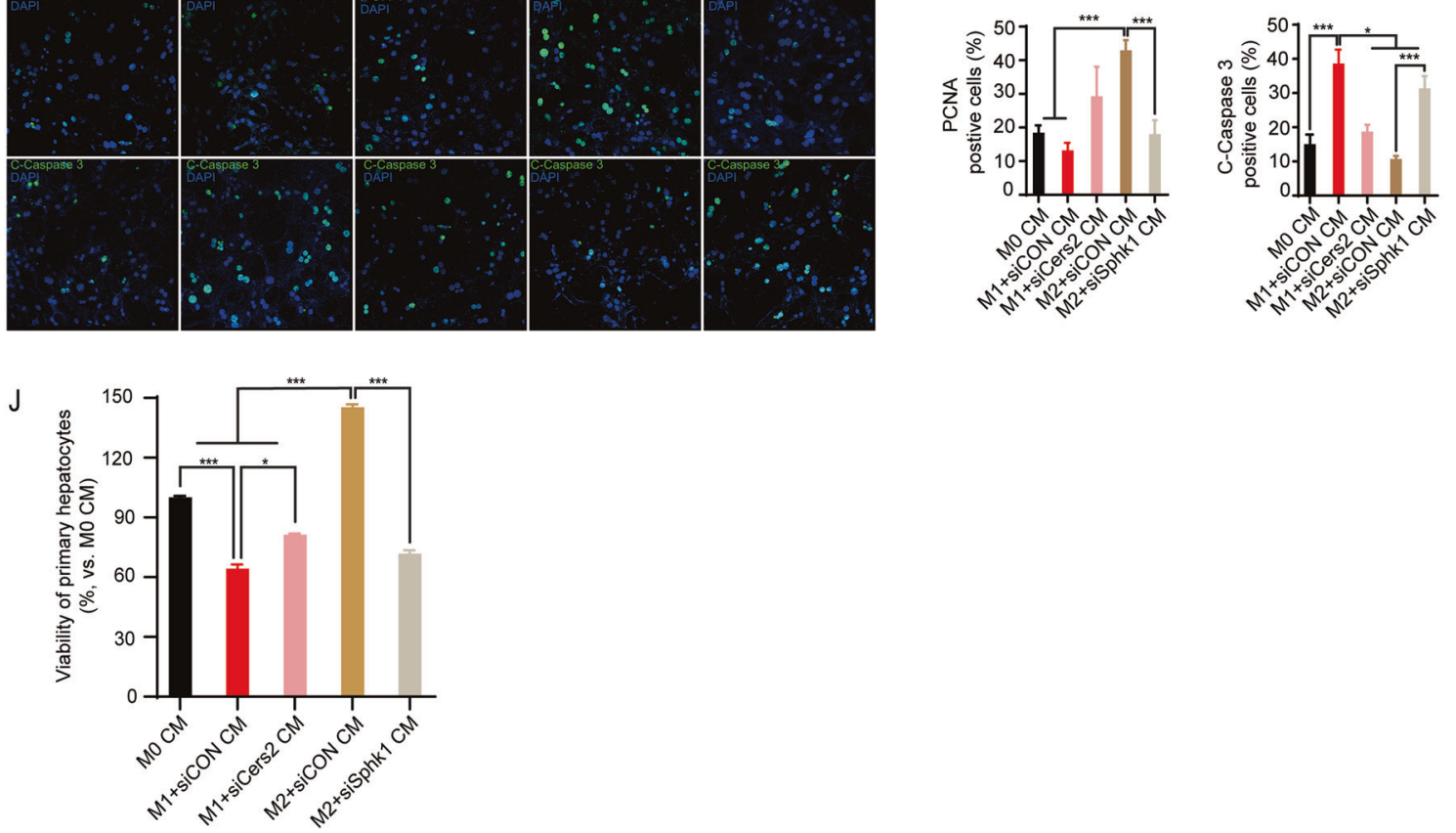

Fig. 6 (See legend on next page.) 
(see figure on previous page)

Fig. 6 Inhibition of CER and S1P synthesis alters the M1- and M2-driven effects on post-hepatectomy hepatocytes, respectively. M1 BMDMs and M2 BMDMs were transfected with siRNA-Cers2 (siCers2) and siRNA-Sphk1 (siSphk1), respectively. A scramble siRNA was used as a control siRNA (siCON). Primary hepatocytes were isolated from the remnant liver at POD2 and incubated with CM from the transfected BMDM, then the proliferation and apoptosis of hepatocytes were examined. A, B Transfection was performed at $24 \mathrm{~h}$ before induction of polarization. mRNA (A) and protein (B) levels of Cers2 and Sphk1 were determined at $48 \mathrm{~h}$ after polarization. C-F BMDMs with siRNA transfection and their medium were collected to measure the levels of CER metabolites, including d18:1-CER (C and E), d18:1-SPH, and d18:1-S1P (D and F). G Hepatocytes were fixed and stained with proliferation marker PCNA and apoptosis maker C-Caspase 3. Top: representative images of immunofluorescence staining for PCNA (green). Bottom: representative images of immunofluorescence staining for C-Caspase 3 (green). Nuclei were counterstained with DAPI (blue). H, I PCNA-positive hepatocytes and C-Caspase 3-positive hepatocytes were enumerated to evaluate the proliferation (H) and apoptosis (I) in hepatocytes. $\mathbf{J}$ The viability of hepatocytes was measured by MTT assay. Images in $\mathbf{B}$ and $\mathbf{G}$ represent results from three independent experiments. Data in $\mathbf{A}, \mathbf{C}-\mathbf{F}$, and $\mathbf{H}-\mathbf{J}$ were demonstrated as mean $\pm \mathrm{SD}, n=3 ;{ }^{*} p<0.05,{ }^{* *} p<0.01,{ }^{* * *} p<0.001$.

mediators for post-hepatectomy liver dysfunction, our study presented that CER and S1P were the critical signature lipids relevant to M1 and M2 polarization in macrophages. Moreover, our data from in vivo and in vitro studies demonstrated that CER and S1P critically mediated the hepatotoxic and hepatoprotective effects of M1- and M2-macrophages infusion on liver recovery after hepatectomy, respectively.

Emerging studies have implicated that bioactive lipids are important signatures of macrophages and play critical roles in mediating the functions of polarized macrophages $^{9,11,13}$. Giannakis et al. reported that resolving D2, a lipid derived from polyunsaturated fatty acids, is a distinct signature of repair macrophages ${ }^{9}$. Montenegro-Burke et al. found that thromboxane A2 derived from arachidonic acid is a specific marker of M1 polarization ${ }^{12}$. In our study, we found that CERs were specifically elevated in response to M1 polarization, whereas $\mathrm{SPH}$, the CERderived lipid metabolites, were found to be increased by M2 polarization (Fig. 1 and Supplementary Fig. 2). With further investigation on the mRNA levels of CER enzymes in M1 and M2 BMDMs, our data further revealed a CERproducing-dominant metabolism in M1 macrophages and a CER-breakdown-dominant metabolism in M2 macrophages (Fig. 2A). Degradation of CER by CDase generates $\mathrm{SPH}$, then SPH is phosphorylated by SPHK to produce $\mathrm{S}^{3} \mathrm{P}^{37}$. Thus, S1P is recognized as the final bioactive product of CER catabolism ${ }^{37}$. Consistent with the upregulation of CER catabolism in M2 BMDMs, our targeted lipidomics data further demonstrated that S1P was substantially elevated by $\mathrm{M} 2$ polarization (Fig. $2 \mathrm{~B}-\mathrm{E}$ ). Intracellular CER, SPH, and S1P have been demonstrated to be secreted extracellularly through lipid vesicles or transporters $^{38,39}$. Therefore, the intracellular increase of CER, $\mathrm{SPH}$, and S1P in polarized macrophages possibly induces the extracellular secretion of CER, SPH, and S1P, resulting in elevation of these lipids in their CM.

Polarized macrophages have recently been implicated in regulating local lipid metabolism ${ }^{29}$. By injection of M1 BMDMs or M2 BMDMs into the remnant liver after hepatectomy, we found that in company with the corresponding alteration in macrophage polarization, M1-BMDM infusion also augmented the CER elevation in the remnant liver after hepatectomy, while M2-BMDM infusion reduced the levels of CER and increasing the levels of SPH and S1P (Fig. 3H, I). These data indicate that the infused polarized macrophages contribute to CER and S1P production in microenvironment of posthepatectomy livers. Interestingly, consistently with in vivo experiments, by treating primary hepatocytes with the CM from polarized BMDMs, we also found that the CER levels or S1P levels were upregulated by the treatment of M1- or M2 CM (Fig. 5E, F). Despite CER and S1P produced by polarized BMDMs may directly enter liver cells to increase the intracellular levels of CER and S1P of liver cells, these data also suggest that polarized macrophages play a role in regulating the CER metabolism in targeted hepatocytes. Although the mechanism is still not clear, previous studies have found that the CER metabolic enzymes on the plasma membrane can be secreted within vesicles, then the vesicles containing CER metabolic enzymes can incorporate into targeted cells to regulate the CER metabolism ${ }^{20}$. Moreover, specific cytokines produced by polarized macrophages have also been found to regulate CER metabolism in targeted cells. For instance, TNF- $\alpha$ is known to upregulate CER synthesis ${ }^{40}$. Therefore, M1 and M2 macrophages may have the potential to directly or indirectly regulate CER metabolism in hepatocytes by incorporating their CERS, CDase, or SPHK into targeted hepatocytes or by producing cytokine through extracellular secretion.

CER and S1P are important bioactive lipids that distinctly regulate various cell biology related to tissue injury and regeneration ${ }^{37}$. CERs are reported as pro-apoptotic lipids. Both intracellular and extracellular accumulation of CER induces cell death by activating the particular apoptotic or necrotic pathway ${ }^{41-43}$. In contrast to CER, S1P is a pro-survival lipid that is crucial in promoting cell proliferation and survival ${ }^{17,18}$. Recent studies demonstrated that extracellular S1P, which was either stored in exosomes or bounded with high-density lipoprotein, was important to promote liver regeneration by enhancing 
angiogenesis and hepatocyte proliferation ${ }^{19,20}$. In line with these studies, we found that elevated hepatic CER induced by M1-BMDM infusion was associated with exaggerated hepatocyte injury after hepatectomy, while increased hepatic S1P induced by M2-BMDM infusion was found to improve liver regeneration after hepatectomy (Figs. 3 and 4). Furthermore, by treating the primary posthepatectomy hepatocytes with exogenous $\mathrm{d} 18: 1 / \mathrm{C}_{16: 0^{-}}$ CER and d18:1-S1P, we found that $\mathrm{d} 18: 1 / \mathrm{C}_{16: 0^{-}}$CER did induce apoptosis, whereas d18:1-S1P treatment promoted proliferation in post-hepatectomy hepatocytes (Fig. 5G, $\mathrm{H})$. Besides bioactive lipids, cytokines produced by the polarized macrophages also play important roles in regulating tissue injury and recovery ${ }^{44,45}$, thus it was more likely that CER and S1P partially mediated the effects of polarized macrophages. We therefore inhibited CER and S1P synthesis in polarized BMDMs by knocking down relevant enzymes, then examined the alteration of M1and M2-driven effects on post-hepatectomy hepatocytes. We found that knockdown of Cers2 inhibited the increase of CER in M1 BMDMs and their CM, and Cers2 knockdown was capable of partially attenuating the pro-death effect of M1 BMDMs (Fig. 6). These data suggested that inhibition of CER synthesis indeed partially attenuated the M1-driven effects. Due to the hydrophobic property of CER, the in vivo delivery system of CER has not yet been established, thus the direct impact of CER on posthepatectomy hepatocytes has not yet been tested. Notably, we found that knockdown of Sphk1 inhibited the increase of S1P and accumulated SPH in M2 BMDMs and their $\mathrm{CM}$, and the CM from Sphk1-knockdown BMDMs promoted cell death in mouse primary hepatocytes (Fig. 6). These data indicated that inhibition of S1P synthesis by Sphk1 knockdown endowed the M2 BMDMs with proapoptotic effects instead of attenuating its pro-survival effects, this might result from the accumulation of proapoptotic $\mathrm{SPH}^{46}$. Notably, consistent with our findings that S1P was important in driving liver recovery from hepatectomy, Nojima et al. have reported that injection of S1P-containing exosomes enhanced liver proliferation ${ }^{20}$. In addition to regulating hepatocyte survival, CER and S1P have also been implicated in regulating macrophage polarization. Our latest study demonstrated that the treatment of unsaturated-long-chain CER promoted the LPS-induced M1 activation on intraperitoneal macrophages $^{47}$. On the other hand, S1P has been reported to promote M2 polarization by enhancing the production of anti-inflammatory cytokines, such as IL- 4 and IL-10 (ref. ${ }^{48-50}$ ). These results suggest that, in addition to regulating apoptosis and proliferation in hepatocytes after hepatectomy, elevation of CER and S1P caused by M1and M2-BMDM infusion may also involve in driving M1 and M2 polarization of macrophages in the remnant liver after hepatectomy, respectively.

\section{Conclusions}

Our study demonstrates for the first time that CER metabolites are important lipid mediators for the polarized macrophages to regulate liver injury and regeneration after hepatectomy. Particularly, our data highlight that M2 macrophage infusion that promotes the generation of hepatoprotective S1P may have cytotherapeutic capacity for post-hepatectomy liver dysfunction.

\section{Acknowledgements \\ We would like to acknowledge Justin Snider and Cungui Mao in the Cancer Center at Stony Brook University, New York, USA, for technical support of sphingolipidomic analyses. We would like to acknowledge the technical support provided from the Central Laboratory of Southern Medical University, Guangzhou, Guangdong, China, for sphingolipidomic analyses.}

\section{Author details}

'Division of Hepatobiliopancreatic Surgery, Department of General Surgery, Nanfang Hospital, Southern Medical University, Guangzhou, Guangdong,

China. ${ }^{2}$ Department of Hepatopancreatobiliary Surgery, The Second Affiliated Hospital of Guangzhou University of Chinese Medicine, Guangzhou,

Guangdong, China. ${ }^{3}$ The First Clinical College, Southern Medical University, Guangzhou, Guangdong, China. ${ }^{4}$ Department of Medicine and Cancer Center, The State University of New York at Stony Brook, Stony Brook, NY, USA. ${ }^{5}$ Department of Radiation Oncology, Nanfang Hospital, Southern Medical University, Guangzhou, Guangdong, China. ${ }^{6}$ Central Laboratory, Southern Medical University, Guangzhou, Guangdong, China. 'Huiqiao Department, Nanfang Hospital, Southern Medical University, Guangzhou, Guangdong, China. ${ }^{8}$ Department of Colorectal Surgery, Shanxi Cancer Hospital, Taiyuan, Shanxi, China

\section{Author contributions}

K.W., H.S., Q.Z., S.S., G.C., H.X., and C.M. participated in research design, analyzed the data, and wrote the manuscript. H.S., G.C., H.X., X.L., H.P., X.C., L.G., Q.L., Y.L., Y.W., X.H., M.L., and P.H. performed the experiments and helped with data analysis. B.Z., J.Q., Q.Z., S.Y., and Z.C. conducted the animal experiments and histology analysis. C.L. and J.S. helped with the lipidomics analyses. K.W., J.Z., and C.L. supervised the study and revised the manuscript. H.S., S.Y., Q.Z., and H.C. performed the experiments and analyzed the data during manuscript revision. K.W., Q.Z., and C.L. designed and supervised the experiments, corrected the interpretation of the data, and revised the manuscript during revision.

\section{Funding}

This work was supported by the National Natural Science Foundation of China (82070642, 81600462, 81803063, and 81800454), the Natural Science Foundation of Guangdong Province (2017A030313684, 2018KJYZ021, and 2018A030313219), the Outstanding Youths Development Scheme of Nanfang Hospital Southern Medical University (2016006), and the Medical Scientific Research Foundation of Guangdong Province of China (A2018049).

\section{Ethics statement}

All animal experiments were carried out following the protocols approved by the Institutional Animal Care and Use Committee of Nanfang Hospital Southern Medical University (NFYY-2017-03).

Conflict of interest

The authors declare no competing interests.

\section{Publisher's note}

Springer Nature remains neutral with regard to jurisdictional claims in published maps and institutional affiliations.

Supplementary information The online version contains supplementary material available at https://doi.org/10.1038/s41419-021-03616-9. 
Received: 8 July 2020 Revised: 9 March 2021 Accepted: 11 March 2021 Published online: 26 March 2021

\section{References}

1. Ray, S., Mehta, N. N., Golhar, A. \& Nundy, S. Post hepatectomy liver failure - a comprehensive review of current concepts and controversies. Ann. Med. Surg. (Lond.) 34, 4-10 (2018).

2. Prodeau, M. et al. An ordinal model to predict the risk of symptomatic liver failure in patients with cirrhosis undergoing hepatectomy. J. Hepatol. 71 920-929 (2019)

3. Golse, N. et al. New paradigms in post-hepatectomy liver failure. J. Gastrointest Surg. 17, 593-605 (2013).

4. Murray, P. J. Macrophage polarization. Annu. Rev. Physiol. 79, 541-566 (2017).

5. Murray, P. J. et al. Macrophage activation and polarization: nomenclature and experimental guidelines. Immunity 41, 14-20 (2014).

6. Elchaninov, A. V. et al. Dynamics of macrophage populations of the liver after subtotal hepatectomy in rats. BMC Immunol. 19, 23 (2018).

7. Melgar-Lesmes, P., Balcells, M. \& Edelman, E. R. Implantation of healthy matrixembedded endothelial cells rescues dysfunctional endothelium and ischaemic tissue in liver engraftment. Gut 66, 1297-1305 (2017).

8. Ma, P. F. et al. Cytotherapy with M1-polarized macrophages ameliorates liver fibrosis by modulating immune microenvironment in mice. J. Hepatol. 67, 770-779 (2017).

9. Giannakis, N. et al. Dynamic changes to lipid mediators support transitions among macrophage subtypes during muscle regeneration. Nat. Immunol. 20, 626-636 (2019).

10. Kang, S. et al. Semaphorin $6 \mathrm{D}$ reverse signaling controls macrophage lipid metabolism and anti-inflammatory polarization. Nat. Immunol. 19, 561-570 (2018).

11. Lovaszi, M. et al. Sebum lipids influence macrophage polarization and activation. Br. J. Dermatol. 177, 1671-1682 (2017).

12. Montenegro-Burke, J. R. et al. Lipid profiling of polarized human monocytederived macrophages. Prostaglandins Other Lipid Mediat. 127, 1-8 (2016).

13. Nojima, H., Freeman, C. M., Gulbins, E. \& Lentsch, A. B. Sphingolipids in liver injury, repair and regeneration. Biol. Chem. 396, 633-643 (2015).

14. Pagadala, M., Kasumov, T., McCullough, A. J., Zein, N. N. \& Kirwan, J. P. Role of ceramides in nonalcoholic fatty liver disease. Trends Endocrinol. Metab. 23, 365-371 (2012).

15. Pewzner-Jung, Y. et al. A critical role for ceramide synthase 2 in liver homeostasis: II. insights into molecular changes leading to hepatopathy. J. Biol. Chem. 285, 10911-10923 (2010).

16. Pewzner-Jung, $Y$. et al. A critical role for ceramide synthase 2 in liver homeostasis: I. alterations in lipid metabolic pathways. J. Biol. Chem. 285 10902-10910 (2010)

17. Kunkel, G. T., Maceyka, M., Milstien, S. \& Spiegel, S. Targeting the sphingosine-1phosphate axis in cancer, inflammation and beyond. Nat. Rev. Drug Discov. 12, 688-702 (2013).

18. Schuchardt, M., Tolle, M., Prufer, J. \& van der Giet, M. Pharmacological relevance and potential of sphingosine 1-phosphate in the vascular system. Br. J. Pharmacol. 163, 1140-1162 (2011).

19. Ding, B. S. et al. HDL activation of endothelial sphingosine-1-phosphate receptor-1 (S1P1) promotes regeneration and suppresses fibrosis in the liver. JCl Insight 1, e87058 (2016).

20. Nojima, $\mathrm{H}$. et al. Hepatocyte exosomes mediate liver repair and regeneration via sphingosine-1-phosphate. J. Hepatol. 64, 60-68 (2016).

21. Mitchell, C. \& Willenbring, H. A reproducible and well-tolerated method for $2 / 3$ partial hepatectomy in mice. Nat. Protoc. 3, 1167-1170 (2008).

22. Ying, W., Cheruku, P. S., Bazer, F. W., Safe, S. H. \& Zhou, B. Investigation of macrophage polarization using bone marrow derived macrophages. J. Vis. Exp. 76, 50323 (2013).

23. Goddard, E. T., Fischer, J. \& Schedin, P. A portal vein injection model to study liver metastasis of breast cancer. J. Vis. Exp. 118, 54903 (2016).

24. Yang, K., Cheng, H., Gross, R. W. \& Han, X. Automated lipid identification and quantification by multidimensional mass spectrometry-based shotgun lipidomics. Anal. Chem. 81, 4356-4368 (2009).
25. Xia, J., Sinelnikov, I. V., Han, B. \& Wishart, D. S. MetaboAnalyst 3.0-making metabolomics more meaningful. Nucleic Acids Res. 43, W251-W257 (2015).

26. Wang, J. R. et al. Improved sphingolipidomic approach based on ultrahigh performance liquid chromatography and multiple mass spectrometries with application to cellular neurotoxicity. Anal. Chem. 86, 5688-5696 (2014).

27. Severgnini, M. et al. A rapid two-step method for isolation of functional primary mouse hepatocytes: cell characterization and asialoglycoprotein receptor based assay development. Cytotechnology 64, 187-195 (2012).

28. Bourgognon, M., Klippstein, R. \& Al-Jamal, K. T. Kupffer cell isolation for nanoparticle toxicity testing. J. Vis. Exp. 102, e52989 (2015).

29. Remmerie, A. \& Scott, C. L. Macrophages and lipid metabolism. Cell Immunol. 330, 27-42 (2018)

30. Martinez, F. O., Gordon, S., Locati, M. \& Mantovani, A. Transcriptional profiling of the human monocyte-to-macrophage differentiation and polarization: new molecules and patterns of gene expression. J. Immunol. 177, 7303-7311 (2006).

31. Kremser, C. et al. Cell-type-specific expression pattern of ceramide synthase 2 protein in mouse tissues. Histochem. Cell Biol. 140, 533-547 (2013).

32. Ferreira, N. S. et al. Regulation of very-long acyl chain ceramide synthesis by acyl-CoA-binding protein. J. Biol. Chem. 292, 7588-7597 (2017).

33. Rohrbach, T., Maceyka, M. \& Spiegel, S. Sphingosine kinase and sphingosine-1phosphate in liver pathobiology. Crit. Rev. Biochem. Mol. Biol. 52, 543-553 (2017).

34. Zheng, $X$. et al. The sphingosine kinase-1 / sphingosine-1-phosphate axis in cancer: potential target for anticancer therapy. Pharmacol. Ther. 195, 85-99 (2018).

35. Maceyka, M. S. H. H. N. SphK1 and SphK2, sphingosine kinase isoenzymes with opposing functions in sphingolipid metabolism. J. Biol. Chem. 44, 37118-37129 (2005).

36. Citterio, D. et al. Hierarchic interaction of factors associated with liver decompensation after resection for hepatocellular carcinoma. JAMA Surg. 151 846-853 (2016).

37. Hannun, Y. A. \& Obeid, L. M. Sphingolipids and their metabolism in physiology and disease. Nat. Rev. Mol. Cell Biol. 19, 175-191 (2018).

38. van Niel, G., D'Angelo, G. \& Raposo, G. Shedding light on the cell biology of extracellular vesicles. Nat. Rev. Mol. Cell Biol. 19, 213-228 (2018).

39. Nijnik, A. et al. The role of sphingosine-1-phosphate transporter Spns2 in immune system function. J. Immunol. 189, 102-111 (2012).

40. Hernandez-Corbacho, M. J. et al. Tumor necrosis factor-alpha (TNFalpha)induced ceramide generation via ceramide synthases regulates loss of focal adhesion kinase (FAK) and programmed cell death. J. Biol. Chem. 290, 25356-25373 (2015)

41. Obeid, L. M., Linardic, C. M., Karolak, L. A. \& Hannun, Y. A. Programmed cell death induced by ceramide. Science 259, 1769-1771 (1993).

42. Arora, A. S., Jones, B. J., Patel, T. C., Bronk, S. F. \& Gores, G. J. Ceramide induces hepatocyte cell death through disruption of mitochondrial function in the rat. Hepatology 25, 958-963 (1997).

43. Jones, B. E., Lo, C. R., Srinivasan, A., Valentino, K. L. \& Czaja, M. J. Ceramide induces caspase-independent apoptosis in rat hepatocytes sensitized by inhibition of RNA synthesis. Hepatology 30, 215-222 (1999).

44. Vannella, K. M. \& Wynn, T. A. Mechanisms of organ injury and repair by macrophages. Annu. Rev. Physiol. 79, 593-617 (2017).

45. Wynn, T. A. \& Vannella, K. M. Macrophages in tissue repair, regeneration, and fibrosis. Immunity 44, 450-462 (2016).

46. Woodcock, J. Sphingosine and ceramide signalling in apoptosis. IUBMB Life $\mathbf{5 8}$, 462-466 (2006).

47. Wang, K. et al. Alkaline ceramidase 3 deficiency aggravates colitis and colitisassociated tumorigenesis in mice by hyperactivating the innate immune system. Cell Death Dis. 7, e2124 (2016).

48. Park, S. J. et al. Sphingosine 1-phosphate induced anti-atherogenic and atheroprotective M2 macrophage polarization through IL-4. Cell. Signal. 26, 2249-2258 (2014)

49. Rodriguez, Y. I. et al. Sphingosine-1 phosphate: a new modulator of immune plasticity in the tumor microenvironment. Front. Oncol. 6, 218 (2016).

50. Weigert, A. et al. Tumor cell apoptosis polarizes macrophages role of sphingosine-1-phosphate. Mol. Biol. Cell 18, 3810-3819 (2007). 